\title{
Ultra-high field transport in GaN-based heterostructures
}

\author{
S.A. Vitusevich ${ }^{1, *}$, S.V. Danylyuk ${ }^{1}$, B.A. Danilchenko ${ }^{2}$, N. Klein ${ }^{1}$, S.E. Zelenskyii ${ }^{2}$, E. Drok ${ }^{2}$, \\ A.Yu. Avksentyev ${ }^{3}$, V.N. Sokolov ${ }^{3}$, V.A. Kochelap ${ }^{3}$, A.E. Belyaev ${ }^{3}$, M.V. Petrychuk ${ }^{1}$, H. Lüth ${ }^{1}$ \\ ${ }^{1}$ Institut für Schichten und Grenzfächen and CNI - Center of Nanoelectronic Systems for Information Technology, \\ Forschungszentrum Jülich, Jülich D-52425, Germany \\ ${ }^{2}$ Institute of Physics, NAS of Ukraine, 03028 Kyiv, Ukraine \\ ${ }^{3}$ V. Lashkaryov Institute of Semiconductor Physics, NAS of Ukraine, 03028 Kyiv, Ukraine \\ ${ }^{*}$ Corresponding author: phone: +49-2461-612345; fax: +49-2461-612470 \\ E-mail address: s.vitusevich@fz-juelich.de
}

\begin{abstract}
This paper describes measurements of the velocity of electrons at electric fields up to $100 \mathrm{kV} / \mathrm{cm}$ in $\mathrm{GaN} / \mathrm{AlGaN}$ heterostructures. In order to avoid the Joule heating effect, a pulse technique with a time sweep of 10-30 ns was used. The experimental results indicate that overheating of the $2 \mathrm{DEG}$ does not exceed $1000 \mathrm{~K}$ in this electric field range and drift velocity as high as $\sim 10^{7} \mathrm{~cm} / \mathrm{s}$ was obtained. Additionally, the low frequency $1 / f$ noise spectra measured for a different bias voltage are analyzed with respect to field-induced contribution of hopping conductivity in $\mathrm{AlGaN}$ barrier region.
\end{abstract}

Keywords: HEMT, TLM, 2DEG, hot electrons, $1 / f$ noise.

Manuscript received 20.09.06; accepted for publication 23.10.06.

\section{Introduction}

Advantages of the GaN-based materials in comparison to the GaAs are the higher intervalley separation and larger optical phonon energy, resulting in larger drift velocities at high electric fields. The latter is promising for high frequency operation of GaN-based devices and introduces novel hot electron relaxation mechanisms. Therefore experimental and theoretical study of high electric field transport in the nitride-based materials and heterostructures are extremely important. In this communication we present results of steady state and pulse measurements of $\mathrm{GaN} / \mathrm{AlGaN}$ heterostructures up to ultra high electric fields accompanied by low frequency noise measurements. Hot electron relaxation processes are analyzed.

\section{Experimental}

The GaN/AlGaN (33\% Al) undoped heterostructures (designed for high electron mobility transistor application and grown by MOCVD on sapphire substrate with AlN buffer layer) of $1.1 \mu \mathrm{m} \mathrm{GaN}$ and $23 \mathrm{~nm}$ AlGaN covered with a $320 \mathrm{~nm} \mathrm{Si}_{3} \mathrm{~N}_{4}$ passivation layer were investigated. The transmission line mode (TLM) patterns of different channel lengths and of the $100 \mu \mathrm{m}$ channel width were fabricated with using standard ohmic contact process. The distance between TLM contacts varied as $1,5,10,20,25$, and $35 \mu \mathrm{m}$. A room temperature mobility of $1250 \mathrm{~cm}^{2} / \mathrm{Vs}$ and sheet carrier density of $1.05 \times 10^{13} \mathrm{~cm}^{-2}$ were obtained in the $2 \mathrm{DEG}$ of the channel by means of the Van der Pauw method. The contact resistance was measured in low-field (ohmic) region and taken into account when calculating the average electric field. The velocity-electric field dependences in GaN/AlGaN heterostructures were obtained by measurements of the current-voltage, $I-V$, characteristics using nanosecond voltage pulses applied to the sample. This pulse regime minimizes the selfheating effects. The measurements were performed in the temperature range $4-300 \mathrm{~K}$.

\section{Results and discussion}

3.1. Low-field transport and low-frequency noise under self-heating conditions

Under steady-state conditions investigation of transport and noise properties should be carried out with taking into account both hot electrons and self-heating effects due to dissipated Joule electric power. The current-electric field characteristics, $I-E$, measured in dc regime (Fig. 1), are strongly nonlinear, although the considered electric field range is still below the fields of well-developed hotelectron regime expected from theoretical predictions [1]. The current noise spectra also reveal considerable 
deviation from a $1 / f$ law predicted in the McWhorter (fluctuations of concentration) [2] or Hooge (mobility fluctuations) [3] models. The main feature of noise spectra is an increase of the $1 / f$ noise level in the low-frequency range together with increasing of applied electric field as shown in Fig. 2. Between the low- and high-frequency $1 / f$ spectral ranges (flat regions in Fig. 2) a transition region (I) appears, which is characterized by a strong deviation from the $1 / f$ behavior. This region (I) is strongly depending on applied electric field. It is remarkable that in the high-frequency interval the normalized noise level is independent of the applied voltage.

In view of the fact that the self-heating is present together with electrical field simultaneously, the analysis of the noise spectra demands separation of temperature and field effects. To solve this problem, we formulate a theoretical model based on (i) heat dissipation and heattransfer modeling in the device and (ii) self-consistent

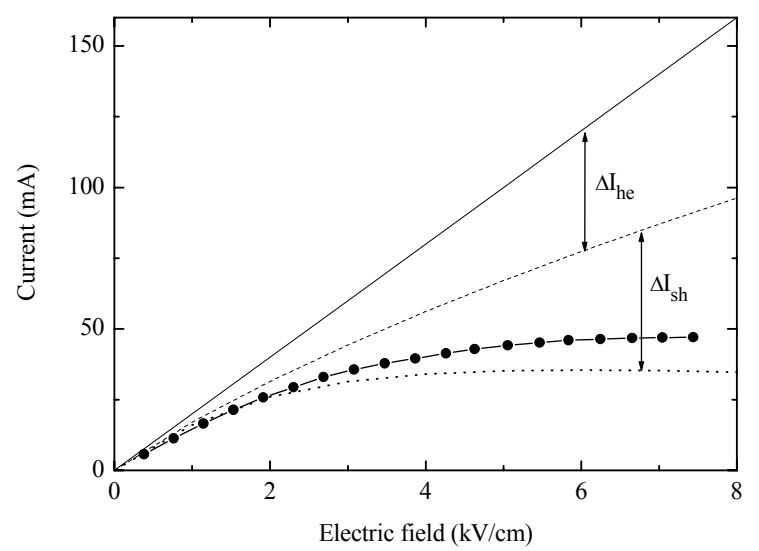

Fig. 1. The result of calculation of $I v s E$ for the device with the channel length of $25 \mu \mathrm{m}$. Solid line is ohmic $I-E$ curve, arrows show current decrease due to hot-electron $\left(\Delta I_{h e}\right)$ and self-heating $\left(\Delta I_{s h}\right)$. Circles the are experimental data measured at $300 \mathrm{~K}$.

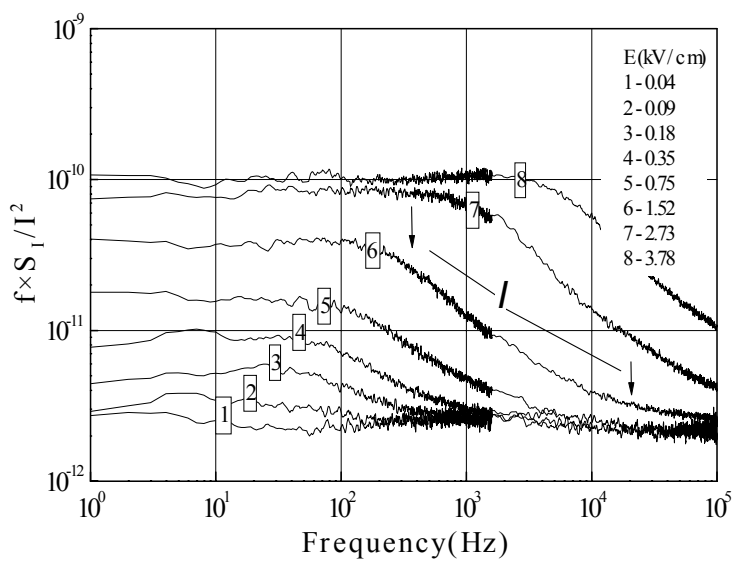

Fig. 2. Spectra of the normalized current noise for different values of the electric field $E$ measured at $T=300 \mathrm{~K}$ for the device with the channel length of $25 \mu \mathrm{m}$. The intermediate region (I) between low- and high-frequency $1 / f$ noise is indicated for the curve 6 . solution of coupled nonlinear equations for the channel current and temperature rise $\Delta T[4]$. The results of simulation for current are presented in Fig. 1. The reasonable coincidence of calculated current to experiment was reached when both contributions (electric field and self-heating) were taken into account. The calculated temperature raise $\Delta T$ versus the dissipated power $P_{\text {dis }}$ in conducting channel is shown in Fig. 3. With respect to obtained results, the temperature rise in noise experiment does not exceed $60 \mathrm{~K}$ at the field $3.78 \mathrm{kV} / \mathrm{cm}$. The latter result allows us to suppose that electric field plays a decisive role in the current noise growth in the region (I), Fig. 2. In assumption that hoping conductivity is present [5], we introduce the phenomenological expression for the current noise for a fixed frequency that could be proportional to

$\Delta J \sim \exp \left(-\frac{\varepsilon_{3}-e V d}{k T}\right)$,

where $\varepsilon_{3}$ is the activation energy of the hoping conductivity, $e$ - electron charge, $V$ - electric field, $d-$ average distance between nearest deep traps, $T$ temperature.

An exponential dependence of $f \times S_{I} / I^{2}$ versus $V$ for two fixed frequencies of $5 \times 10^{3}$ and $1 \times 10^{4} \mathrm{~Hz}$ is clearly seen from Fig. 4 with a slope equal to $e d / k T$. The slope allows to estimate the value of $d$ to be about $5 \times 10^{-6} \mathrm{~cm}$. Such a value is reasonable for the undoped AlGaN barrier layer and considered noise behavior could be attributed to processes of hopping conductivity though deep traps in the layer [5].

\subsection{High-field transport and velocity of electrons in GaN/AlGaN heterostructures}

The investigation of high-field electron transport requires minimization of self-heating by using pulse techniques. In this case, we can study pure hot-electron effects. To determine the electron velocity, $v$, we used the relation $v=I /$ en $W$, where $I$ is the current flowing

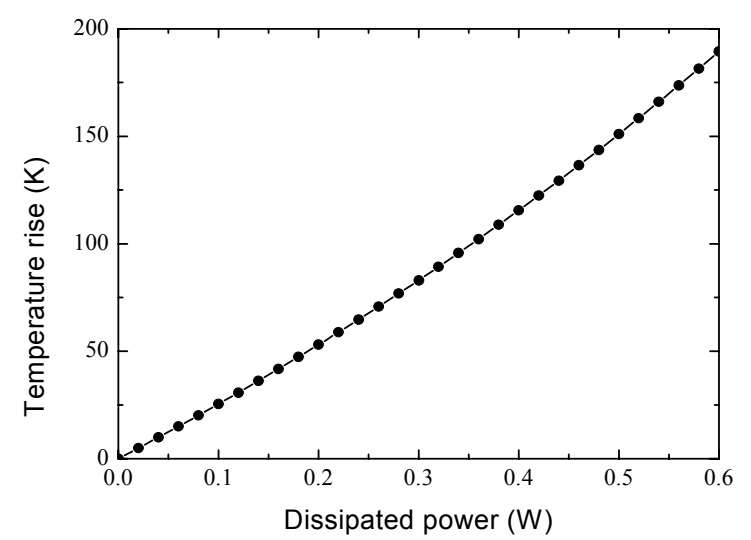

Fig. 3. Dependence of the temperature rise $\Delta T$ due to Joule heating on the dissipative power $P_{\text {dis }}$ in the channel of the $25-\mu \mathrm{m}$ length at the initial temperature $T_{0}=300 \mathrm{~K}$. 


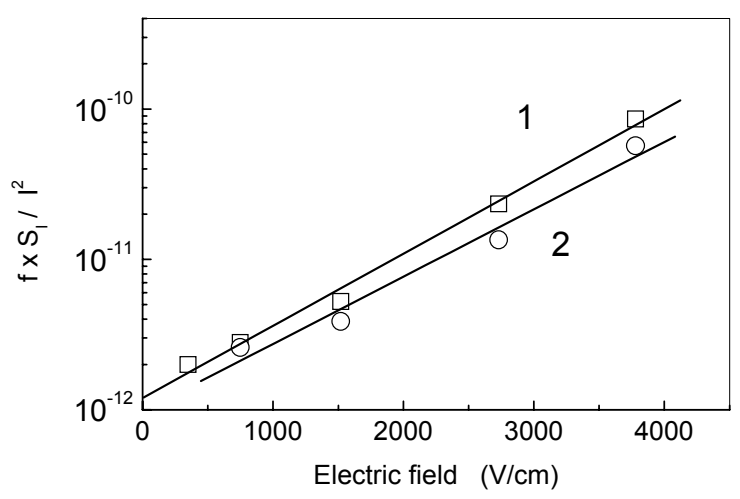

Fig. 4. Dependences of the current noise on the electric field for two fixed frequencies: $5 \times 10^{3}(1)$ and $10^{4} \mathrm{~Hz}(2)$.

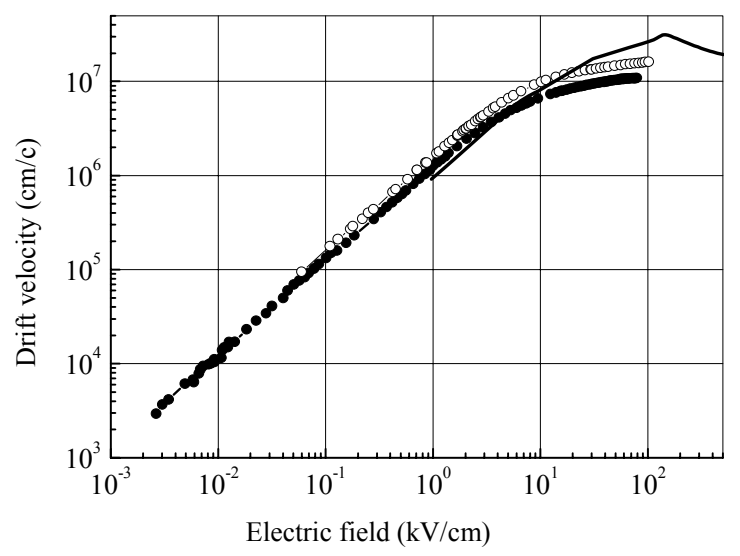

Fig. 5. Electron velocity for two TLM samples as a function of the electric field. The open symbols correspond to $L=$ $=5 \mu \mathrm{m}, T_{0}=4.2 \mathrm{~K}$. The solid symbols correspond to $L=$ $=10 \mu \mathrm{m}, T_{0}=300 \mathrm{~K}$, the solid curve represents the calculated dependence.

within a short pulse (10-30 ns) of applied voltage, $e$ is the electron charge, $n$ is the carrier concentration and $W$ is the width of the conducting channel. This type of measurement relies on the assumption that the carrier concentration remains constant during the measurement even of the electric field changes. The experimental $v-E$ curves (there $E=V / L$ is the average electric field in the conducting channel) measured on TLM with different length are very similar. As it can be seen in Fig. 5, a linear increase in the velocity up to the electric fields of about $5 \mathrm{kV} / \mathrm{cm}$ is followed by a sub-linear dependence. It is remarkable that despite our nitride structures have relatively small low-field electron mobility (4000 and $1250 \mathrm{~cm}^{2} / \mathrm{Vs}$ at 4.2 and $300 \mathrm{~K}$, respectively) we obtain the magnitude of the drift velocity above $10^{7} \mathrm{~cm} / \mathrm{s}$ that is very close to that predicted by the theory [1].

Because of the large carrier concentration, the electron-electron (e-e) scattering dominates over other relaxation mechanisms, and the shifted Maxwellian function with an effective electron temperature $T_{e}$ and a

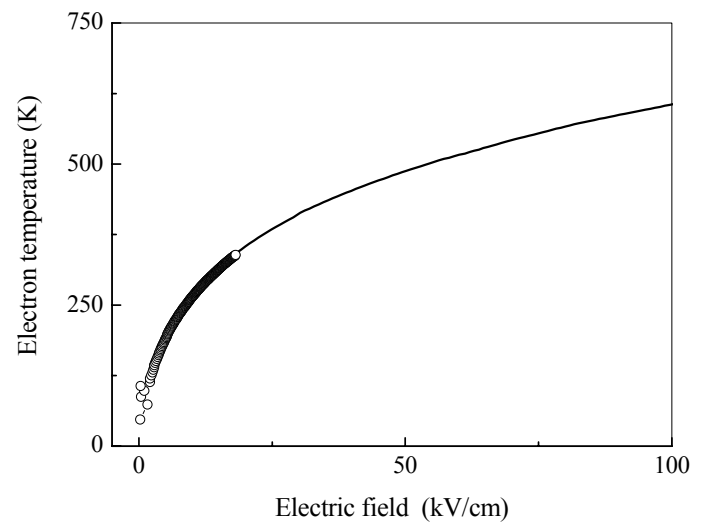

Fig. 6. Electron temperature $T_{e}$ dependence on the electric field. The circles are experimental data; the solid curve represents an extrapolation to high electric fields.

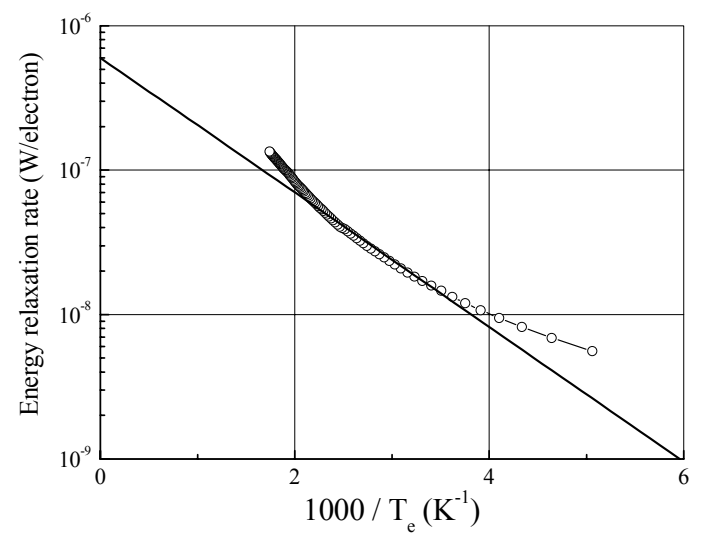

Fig. 7. Dependence of the electron energy relaxation rate $P_{e}$ (in $\mathrm{W}$ per electron) on the inverse electron temperature. Circles are the experimental data, solid line is the fitting of Eq. (2) with $E=92 \mathrm{meV}$ and the fitting parameter $\tau=25 \mathrm{fs}$.

drift velocity provides good approximation for the electron distribution [6]. $T_{e}$ can be estimated by the "mobility comparison" method [7]. It is clearly seen in Fig. 6, where experimental points are plotted together with extrapolation toward high electric fields that even at $E=100 \mathrm{kV} / \mathrm{cm} T_{e}$ does not exceed $650 \mathrm{~K}$. To analyze the sources of the energy relaxation by electrically heated electrons, we plotted the energy relaxation rate per electron against the inverse electron temperature. It is clearly seen from Fig. 7 that in the temperature range $250 \mathrm{~K}<T_{e}<500 \mathrm{~K}$ optical phonon emission is the dominant energy relaxation process. In this case, the standard expression for the electron energy relaxation rate should be used [7]:

$$
P_{e}=\frac{\hbar \omega_{\mathrm{LO}}}{\tau} \exp \left(-\frac{\hbar \omega_{\mathrm{LO}}}{k T_{e}}\right),
$$

where $\hbar \omega_{\mathrm{LO}}$ is the LO phonon energy and $\tau=\left(2 \alpha \omega_{\mathrm{LO}}\right)^{-1}$ is the electron-LO phonon scattering 
time, $\alpha$ is the Fröhlich coupling constant. In the mentioned temperature range, the experimental data are very close to the calculated ones with $\hbar \omega_{\mathrm{LO}}=92 \mathrm{meV}$, which is well known, and $\tau=25 \mathrm{fs}$ as fitting parameter. It should be noted out that the estimated values of $T_{e}$ are much lower (at $E=90 \mathrm{kV} / \mathrm{cm}, T_{e}$ estimated from our experiments is about $600 \mathrm{~K}$ ) then those determined by measurements of the noise temperature, where for the same value of the electrical field the noise electron temperature $T_{e}$ is about $1150 \mathrm{~K}$ [8].

\section{Conclusions}

In this paper, we presented experimentally measured low and high field transport phenomena for a twodimensional electron gas in GaN/AlGaN heterostructure. Under steady-state conditions, investigation of transport and noise properties have been carried out with taking into account both hot electrons and self-heating effects due to the dissipated Joule electric power. Noise increasing with an applied voltage is assumed to be the field induced hopping conductivity in AlGaN barrier.

In ultra short pulse regime, we obtained the magnitude of the drift velocity above $10^{7} \mathrm{~cm} / \mathrm{s}$ that is very close to that predicted by the theory. Estimation of thermal budget of the system shows that overheating of the $2 \mathrm{DEG}$ does not exceed $650 \mathrm{~K}$ up to electric fields about $100 \mathrm{kV} / \mathrm{cm}$.

\section{Acknowledgements}

The authors would like to thank V. Tilak, J. Smart, A. Vertiatchikh and L.F. Eastman (Cornell University) for their collaboration in this study. This work is supported by the Office of Naval Research under Grant No. N00014-01-1-0828 (Project Monitor Dr. Colin
Wood) and by Deutsche Forschungsgemeinschaft (project No. KL 1342/3). The work at Institute of Semiconductor Physics in Kyiv was supported by CRDF Project No. UE2-2439-KV-02 and Institute of Physics by Ukrainian FFR Project F7/379.

\section{References}

1. E.A. Barry, K.W. Kim, V.A. Kochelap, Hot electrons in group-III nitrides at moderate electric fields // Appl. Phys. Lett. 80(13), p. $2317-9$ (2002).

2. Sh. Kogan, Electronic noise and fluctuations in solids. Cambridge University Press, Cambridge, UK, 1996.

3. F.N. Hooge, T.G.M. Kleinpenning, L.K.J. Vandamme, Experimental studies on $1 / \mathrm{f}$ noise // Repts Progr. Phys. 44(5) p. 479-532 (1981).

4. S.A. Vitusevich, S.V. Danylyuk, N. Klein et al., Separation of hot-electron and self-heating effects in two-dimensional $\mathrm{AlGaN} / \mathrm{GaN}$-based conducting channels // Appl. Phys. Lett. 82(3), p. 748-750 (2003).

5. D.C. Look, D.C. Reynolds, W. Kim, O. Aktas, A. Botchkarev, A. Salvador, and H. Morkoc, Deepcenter hopping conduction in GaN // J. Appl. Phys. 80(5), p. 2960-2963 (1996)

6. P. Tripathi, B.K. Ridley, Dynamics of hot-electron scattering in GaN heterostructures // Phys. Rev. B 66(19), 195301-10 (2002).

7. N.M. Stanton, P. Hawker, A.J. Kent, T.S. Cheng, and C.T. Foxon, Hot electron energy relaxation in gallium nitride // Phys. status solidi (a) 176(1), p. 369-372 (1999).

8. A. Matulionis, J. Liberis, I. Matulioniene et al., Hot-phonon temperature and lifetime in a biased $\mathrm{Al}_{\mathrm{x}} \mathrm{Ga}_{1-\mathrm{x}} \mathrm{N} / \mathrm{GaN}$ channel estimated from noise analysis // Phys. Rev. B 68(30) 035338-1-7 (2003) . 